\title{
Literaturwissenschaft
}

\section{Barbara Rowińska-Januszewska}

ORCID: 0000-0002-5253-2530

Poznań

DOI: $10.19195 / 0435-5865.143 .1$

\section{Zum Bild von Angelika Kauffmann in Alioths Der prüfende Blick}

\begin{abstract}
s
Der folgende Text untersucht das literarische Bild der Malerin Angelika Kauffmann im historischen Künstlerroman Der prüfende Blick (2007) von Gabrielle Alioth. Die Schweizer Autorin präsentiert in ihrem intermedialen Buch ein multiperspektivisches Vexierbild der Malerin aus dem 18. Jh., die als eine der ersten Frauen den beruflich-künstlerischen Weg erfolgreich realisieren konnte. Alioth bietet ein vielfältiges, literarisch-malerisches Porträt der Künstlerin aus der Sicht von ihren Freunden und Bekannten, das auch ihre Malerei beinhaltet.
\end{abstract}

Schlüsselwörter: Schweizer Frauenliteratur, Malerei in Literatur, Angelika Kauffmann

\section{Image of Angelika Kauffmann in Der prüfende Blick by Alioth}

The following study examines the literary image of the painter Angelika Kauffmann in the historical novel Der prüfende Blick (2007) by Gabrielle Alioth. In her book, the Swiss author presents a multiperspectival picture of the painter from the 18th century, who was one of the first women to successfully pursue the artistic path. Alioth offers a diverse image of the artist from the perspective of her friends and acquaintances, including her paintings.

Keywords: Swiss female literature, painting in literature, Angelika Kauffmann

Barbara Rowińska-Januszewska, Os. Dębina 19/28, 61-450 Poznań, Polen, E-Mail: barrov@wp.pl Received: 24.09.2017, accepted: 4.05.2018 
...Jedes unserer Bilder zeigt nicht nur das, was wir sehen, sondern spiegelt auch, was wir sind. (Blick 235)

Im Jahre 2007 erschien der historische Künstlerroman Der prüfende Blick. Roman über Angelica Kauffmann von Gabrielle Alioth, die seit 1984 die meiste Zeit über in Irland lebt. Neben Eveline Hasler gehört Alioth zu den wichtigsten Deutschschweizer Autoren von historischen Romanen. ${ }^{1} \mathrm{Zu}$ ihren historischen Werken gehören Der Narr, Wie ein kostbarer Stein, Die Braut aus Byzanz oder Die griechische Kaiserin. In zeitgenössischen Büchern greift sie die Problematik der Liebe und Beziehungen auf (Die Arche der Frauen, Die Erfindung von Liebe und Tod) oder entwirft spannende Detektivgeschichten (Die entwendete Handschrift). Sie schreibt ebenfalls Sach- (Ausgewandert: Schweizer Auswanderer aus 7 Jahrhunderten) und Reisebücher, Kinderbücher sowie Theaterstücke (Orpheus!). Wie die passionierte Schriftstellerin konstatiert, interessieren sie „Geschichten, die uns betreffen, Geschichten von Suchen und Finden, Trennung und Wiedersehen, Liebe und Tod" (Alioth: Schreibinseln 2007: 57), wobei sie sich auf Rekonstruktion von alten Stoffen und Motiven spezialisiert. ${ }^{2}$ Sie scheut aber nicht davor zurück, sich zu aktuellen politisch-gesellschaftlichen Fragen zu äußern (z.B. über EU).

Alioths Der prüfende Blick, zu dem die Autorin umfangreiche Recherchen betrieb (s. Alioth 2015), gehört neben den Romanen Valerie oder Das unerzogene Auge von Erica Pedretti, Das geheime Fieber von Christoph Geiser oder Ombra von Urs Faes zu den wichtigsten deutschsprachigen Werken aus der heutigen Schweiz, in denen die Malerei und das Künstlerleben von großer Relevanz sind. ${ }^{3}$ Es gibt mehrere Möglichkeiten, die Kunst in ein literarisches Werk aufzunehmen: Dargestellt wird ein Besuch im Museum, beschrieben beziehungsweise erwähnt wird ein Gemälde, zu den Protagonisten gehört ein fiktiver respektive authentischer Maler oder Kunstkenner. Alioth wählt zu ihrer Hauptfigur eine au-

${ }^{1}$ Zu Alioths historischen Romanen s. Siobhán Donovan: Dichtung und Wahrheit, Fiktion und Rekonstruktion: Zur Metamorphose der griechischen Kaiserin Theophanu. In: Pender / Burns 2015: 19-32; Kunze 2008.

${ }^{2}$ Vgl. dazu Gabrielle Alioth: Das Märchen von der Zukunft. In: DerStandard.at 20.06.2016, http://derstandard.at/2000039290670/Gabrielle-Alioth-Das-Maerchen-von-der-Zukunft (Zugriff am 7.04.2018).

${ }^{3} \mathrm{Zu}$ erinnern sei hier an Gottfried Kellers Der grüne Heinrich, dessen Held Heinrich Lee von Beruf Maler war. Keller beschäftigte sich auch mit der Malerei, ähnlich wie die Schweizer Schriftsteller Silvio Blatter oder Erica Pedretti. Zur Malerei in der Deutschschweizer Literatur s. Barbara Rowińska-Januszewska: ,, Der unfertige Himmel “ - Malerei und Freundschaft in „,Ombra “von Urs Faes. In: Dies.: Im helvetischen Literaturraum. Schneetod und andere Untersuchungen. Poznań 2017, S. 205-206. 
ßergewöhnliche, authentische Persönlichkeit aus der Künstlerwelt des 18. Jahrhunderts, eine klassizistische, österreichisch-schweizerische ${ }^{4}$ Malerin und ihre Werke, die einen integralen Teil des Romans konstituieren. Neben Kauffmann sind im Buch auch Werke von anderen Künstlern präsent, wie Raphael, Michelangelo, Mengs, Füssli oder Reynolds.

In Der prüfende Blick, ${ }^{5}$ der zum 200. Todestag von Angelika Kauffmann ${ }^{6}$ (1741-1807) erschien, ${ }^{7}$ präsentiert Alioth ein facettenreiches, multiperspektivisches Porträt der vielseitig begabten und gut ausgebildeten Malerin und ihrer Epoche, die als erste deutschsprachige Künstlerin vom europäischen Rang in

${ }^{4}$ Angelikas Mutter Cleophea Lutz war Schweizerin und stammte aus Chur, ihr Vater Johann Kauffmann stammte aus Vorarlberg, aus Schwarzenberg.

5 Zürich 2007. Der Roman wird weiterhin als „Blick“ verzeichnet. Außer Rezensionen erschien bisher kein längerer Beitrag über den Roman. Malcolm Pender berücksichtigt den Roman in seinem Artikel „, Man kann das Gegenwärtige nicht ohne das Vergangene erkennen “als eins von drei Büchern. Romane über Kauffmann verfassten auch Anne Thackeray (auf Englisch: Miss Angel, 1875), Hilde Passow-Kernen (Künstlerroman Angelika Kauffmann. Zürich 1942), Jutta Rebmann, Simona Weller (Ital. Ritratto di Angelica, dt. Übers. v. Christel Galliani: Die zehnte Muse. Das Leben der Angelika Kauffmann. Roman. München 1999) oder Ursula Naumann (Geträumtes Glück. Angelica Kauffmann und Goethe, 2007).

${ }^{6}$ Es sei zu bemerken, dass man immer noch verschiedene Schreibweisen von Angelika Kauffmanns Vornamen und Namen verwendet - zuletzt überwiegt die Tendenz, den Vornamen mit „,k“ $\mathrm{zu}$ schreiben. Alioth verwendet im ganzen Roman die ältere Schreibweise mit „c“. Kauffmann gehört neben Anna Dorothea Therbusch-Lisiewska (1721-1782), Caroline Bardua, Ludovike Simanowitz (Porträts von Schiller), Marie Ellenrieder (1791-1861, die einzige Frau unter Nazarenern) oder Louise Seidler (Goethes Vertraute) zu den ersten und bedeutendsten deutschsprachigen Malerinnen. Zugleich ist sie - neben Jean-Étienne Liotard, J.H. Füssli, Anton Graff, Caspar Wolf, Ferdinand Hodler, Arnold Böcklin, Paul Klee, Segantini oder Giacometti eine der wichtigsten Vertreterinnen der Malerei aus der Schweiz. Zur Schweizer Malerei s. Bernadetta Matuszak-Loose: Malowanie „nieba bez ojczyzny“. Rzecz o osobliwościach sztuki szwajcarskiej. In: Barbara Rowińska-Januszewska: Między „rajem“ a ,więzieniem“. Studia o literaturze i kulturze Szwajcarii. Poznań 2004, S. $163-177$.

${ }^{7}$ Im selben Jahr erschienen auch das Buch von Ursula Naumann Geträumtes Glück. Angelica Kauffmann und Goethe, die Neuaufl. vom beachtenswerten, biographischen Roman von Jutta Rebmann Angelika Kauffmann. Gefeierte Malerin im Europa des 18. Jahrhunderts. Biographischer Roman (1. Ausg. Mühlacker 1994 - mit Bibliographie und Zeittafel, Kurzbiographien von wichtigsten Persönlichkeiten) oder Tobias G. Natter (Hrsg.): Angelika Kauffmann. Ostfildern 2007 [Für die Ausstellung Angelika Kauffmann: Ein Weib vom ungeheuren Talent im Vorarlberger Landesmuseum, Bregenz u. Angelika-Kauffmann-Museum, Schwarzenberg, vom 14. Juni bis 5. Nov. 2007].

Germanica Wratislaviensia 143, 2018

(C) for this edition by CNS 
die Kunst- und Kulturgeschichte einging. ${ }^{8}$ Nach einer Periode des Überdrusses 9 und gewisser Vergessenheit ${ }^{10}$ gilt Angelika Kauffmann heutzutage als eine der wichtigsten Frauen in der Kunstgeschichte. ${ }^{11}$ Sie reüssierte als berühmte Porträtistin und Historienmalerin in London und Rom, verkehrte in Kreisen von angesehenen Künstlern, Dichtern und Gelehrten der damaligen Zeit. Alioth präsentiert im Roman mehrere Bildnisse und Gemälde der Kunstschaffenden, ${ }^{12}$ wodurch der Leser gleichsam dazu gezwungen wird, sich gleichzeitig die Bilder der Malerin anzuschauen und mitzurezipieren. Auf diese Weise wird die Lektüre dieses intermedialen Romans zum literarisch-visuellen, ästhetischen Erlebnis, Alioths Buch dagegen - zum literarisch-malerischen Bildnis der Künstlerin. Trotz vieler Bild-

${ }^{8}$ Kauffmann hinterließ über 600 Gemälde mit verschiedenen Motiven. Von ihrem Erfolg zeugt u.a. die Tatsache, dass schon drei Jahre nach ihrem Tod ihre erste Biographie von Giovanni Cherardo Rossi: Vita di Angelica Kauffmann (Florenz 1810/Bregenz 1814, ins Deutsche üb. u. erläutert v. Alois Weinhart: Wien 1866) erschien. Rossi, Gerard, Engels wie auch andere Biographen berufen sich auf Angelikas Schwager Giuseppe Carlo Zucchi, der seit 1782 Materialien und Informationen zu Angelika Kauffmann und ihrem Werk systematisch sammelte (er machte handschriftlich detailreiche Notizen). Ein Kapitel widmet ihr Alexander von Sternberg in: Berühmte deutsche Frauen des achtzehnten Jahrhunderts (1848), sowie Ida von Düringsfeld in: Das Buch denkwürdiger Frauen. Lebensbilder und Zeitschilderungen (1877). Richard Muther schreibt über Kauffmanns Malerei im 5. Bd. seiner Die Geschichte der Malerei. Leipzig 1906, S. 154-155. Marburger Kunstgeschichtsprofessor Richard Hamann schrieb über sie in Die deutsche Malerei im 19. Jahrhundert. Leipzig 1913, S. 37) sowie in Die deutsche Malerei. Vom 18. bis zum Beginn des 20. Jahrhunderts (Leipzig/Berlin 1952, S. 74). Von den letzten Werken über Kauffmann sei hier auf die Monographie von Waltraud Maierhofer, Veröffentlichungen von Bettina Baumgärtel (Diss. über Kauffmann), die englischsprachige Biographie von Angelica Goodden, oder die Biographie der Kunsthistorikerin Gabriele Katz zu verweisen.

${ }^{9}$ Es hing damit zusammen, dass Kauffmanns Bilder in Form von Kupferstichen, Radierungen, zahlreichen Kopien sowie Porzellan- und Möbelbildern weit verbreitet waren (s. Lettau 1998, auch Goodden 2005: 10).

${ }^{10}$ Kauffmann geriet für gewisse Zeit in Vergessenheit, und zwar so sehr, dass sie im Album Schweizer Maler. 100 Bilder des Schweizerischen Beobachters (Hrsg. vom Beobachter-Verlag. Glattbrugg 1976) nicht berücksichtigt wurde. Dasselbe gilt für die dreibändige Dzieje sztuki w zarysie v. Ksawery Piwocki (Warszawa: Arkady 1977). Dagegen berücksichtigt Maria Rzepińska Kauffmann neben Mengs in Siedem wieków malarstwa europejskiego (Warszawa 1986).

${ }^{11}$ Gottfried Sello berücksichtigt sie neben 46 anderen Künstlerinnen in: Malerinnen aus vier Jahrhunderten. Hamburg 2004. Sie wird häufig neben der franz. Malerin Elisabeth Vigée-Lebrun erwähnt.

${ }^{12}$ Was etwas verwundern mag, berücksichtigt die monumentale, wertvolle, enzyklopädische Arbeit von Konstanze Fliedl / Marina Rauchenbacher / Joanna Wolf (Hrsg.): Handbuch der Kunstzitate: Malerei, Skulptur, Fotografie in der Deutschsprachigen Literatur der Moderne. Berlin-Boston 2011 (mit zahlreichen Abbildungen) Alioths Roman nicht. Im Handbuch der Kunstzitate werden viele helvetische Schriftsteller erfasst, was von ihrem großen Interesse an der Kunst zeugt. Zu verweisen sei hier auch auf Konstanze Fliedl / Bernhard Oberreither / Katharina Serles (Hrsg.): Gemälderedereien. Zur literarischen Diskursivierung von Bildern. Berlin 2013; oder G. Królikiewicz / O. Płaszczewska / I. Puchalska / M. Siwiec (Hrsg): Literatura a malarstwo-malarstwo a literatura. (Kraków 2009), wo gegenseitige Wechselwirkungen zwischen Literatur und Malerei untersucht werden. 
beschreibungen und Kunstzitate ${ }^{13}$ steht im Zentrum des Interesses von Alioth die Malerin selbst, ihr Wesen, Charakter und ihre Faszinationen. Kauffmann war nicht nur eine der talentiertesten Malerinnen in der Geschichte, sie war auch musikalisch begabt, wohl belesen und gehörte zu den intelligentesten Frauen ihrer Epoche. Als eine der ersten Frauen, die beruflich ein erfülltes und erfolgreiches Leben führten, kann sie als Vorläuferin der Frauenbewegung gelten.

Alioth verwendet in ihrer literarischen Künstlerbiographie, die auch als Annäherungsversuche an Angelika Kauffmann betitelt werden könnte, eine etwas ungewöhnliche Komposition und Erzählperspektive und zeigt ihre Protagonistin indirekt, ausschließlich aus der Perspektive von einigen Personen aus ihrem Bekanntenkreis. Sie wählt dabei meistens authentische Figuren, wie Füssli, Morgan, Reiffenstein oder Zucchi und verbindet gekonnt das Faktische mit dem Fiktiven. ${ }^{14}$ Daraus entsteht zuweilen auch ein verwirrendes, vielseitiges Puzzle- oder Suchbild der Malerin: Das Ausmaß des Materials und viele Nebenexkurse mögen überwältigend wirken, ebenso enttäuschend kann die Absenz von Kauffmann erscheinen. ${ }^{15} \mathrm{Um}$ das Buch richtig zu verstehen und sich in der Fülle von Informationen, Personen und Begebenheiten nicht zu verlieren, muss man die Biographie Kauffmanns kennen ${ }^{16}$ und bei der Lektüre - wie angedeutet - die Bilder der Malerin gleichsam visuell mitverfolgen. Die Handlung des anspruchsvollen Romans spielt um 1800 im Künstlermilieu: Der Ich-Erzähler Franz Joseph Kauffmann ist Landschaftsmaler und der Verwandte der Malerin, der vor vierzig Jahren Lehrling ihres Vaters, des Wandermalers Johann Kauffmann (1707-1782) war. Er verbrachte mit Angelika seine Jugend und arbeitete mit ihr zusammen - unter Leitung ihres Vaters, eines mittelmäßigen Malers, aber talentierten Lehrers - in der Kirche in Schwarzenberg (an Gemälden und Fresken) sowie im Schloss in Tettnang. Lange Zeit in sie verliebt, zeigt er sich selbst nach vielen Jahren weiterhin von ihr und ihren Werken fasziniert. Der Aschaffenburger Landschaftsmaler und ehemaliger Hofmaler in Mainz, mittlerweile glücklich verheiratet und Vater von zwei Kindern, erzählt von Angelikas künstlerischem Werdegang, ihren Erfolgen und Niederlagen in Form von Erinnerungen, Rückblenden, Briefen, Tagebuchaufzeichnungen oder Gesprächen mit ihren Bekannten und Verwandten. Stellenweise

${ }^{13}$ Verweise auf authentische Kunstwerke sind als Kunstzitate zu betrachten. S. Einleitung in Handbuch der Kunstzitate. Mit Kunstzitaten wird gleichsam an das Erinnerungs- und Imaginationsvermögen des Lesers appelliert, das Zusammenspiel von Wort und Bild bereichert den literarischen Text um zusätzliche Dimensionen.

${ }^{14}$ Vgl. (Alioth 2015: 12): „Die Lücken, die zwischen den Fakten zwangläufig bleiben [...], fülle ich mit Fiktion".

15 Diese Perspektive, in der Alioth ihre Protagonistin selbst nicht zu Wort kommen lässt, sowie viele „Nebenporträts“ (z.B. über Mengs, Winckelmann, Füssli, Bodmer, Lavater, B. West, Reynolds, Hone oder Morgan), Digressionen und Abschweifungen vom Hauptthema werden von Rezensenten (s. Birrer 2007, Hammer 2007) kritisiert. Eva Bachmann bemängelt die große Anzahl von Personen, Namen und Details, wodurch der Überblick leicht verlorengehen kann.

16 Dies vermerkt mit Recht Klaus Hammer in seiner Rezension Auf der Spur einer Suchfigur. 
übernimmt er die Rolle einer Instanz von oben, korrigiert und kommentiert die Erzählungen seiner Gesprächspartner. Mehrmals stellt er sich die Frage nach dem Wesen, Charakter und Persönlichkeit der heutigen Angelika. Als Ausgangspunkt seiner Erzählung und Reise nach Schwarzenberg, ${ }^{17}$ Bregenz und Venedig wird ihm der Wunsch, seiner malerisch talentierten Tochter Katharina eine Lehrstelle bei der berühmten Cousine zu besorgen.

In einzelnen Kapiteln werden wichtigste Stationen aus Angelikas Leben, ihre Persönlichkeit und Interaktionen mit anderen Menschen, sowie ihre Malerei aus mehreren Seiten belichtet, wobei Alioth eine Art Spiegel- oder „Kristall“-Perspektive verwendet: „Wie ein Kristall hat sie sich in den Erinnerungen von Füssli, Morgan, dem Sänger, dem Pfarrer vor meinen Augen gedreht und immer wieder das Bild des Betrachters zurückgeworfen“" (vgl. Blick 138). So schildert der alte, blinde Pfarrer aus Schwarzenberg ihre Kindheit und Anfänge als Künstlerin, als sie pflichtbewusst schon als sechzehnjährige an den Fresken und Bildern in der Kirche arbeitete. Damals wurde Angelika von der Familie von Salis sowie vom Bischof Nevroni unterstützt, der das Talent des „Wunderkindes“18 früh erkannte, sein Bildnis bei junger Malerin bestellte und ihr künstlerisches Talent anderen Bischöfen und Priestern empfahl. Dadurch bekam nicht nur ihr Vater, sondern auch sie neue, gut bezahlte Aufträge.

Kauffmann war nicht nur malerisch, sondern auch musikalisch talentiert: Mit ihrem wunderbaren, weichen Sopran hätte „,sie [...] eine berühmte Sängerin werden können, bewundert, geliebt [...]“ (Blick 45). Nach dem Sänger und Opernkomponisten von Tettnang war „,das Mädchen im Malerkittel“ (Blick 47) ein außergewöhnliches, musikalisch talentiertes Wesen, das er für seine Oper über Eurydike und Orpheus engagierte und in ihren wenigen freien Stunden ${ }^{19}$ trainierte. Er nannte sie Eurydike und sah im Mädchen „mit Pinsel und Palette“ (Blick 61) eine höchst musikalisch und schauspielerisch begabte Schülerin, die imstande wäre, verschiedene große Opernpartien und -rollen zu übernehmen. Auf der Opernbühne hätte sie ,[...] alles werden können, was sie sich erträumte: Ariadne, Iphigenie, Penelope, Diana oder Venus“ (Blick 53). ${ }^{20}$ Nach seiner Auffassung war „Musik [...] ihre Bestimmung“ (Blick 64). Dass Musik keine vorübergehende Schwärmerei der Malerin war, zeigt Kauffmanns erstes, stilisiertes Selbstbildnis als Sängerin mit Notenblatt ${ }^{21}$ (um 1753-Blick 5), ihr berühmtes Selbstbildnis am Scheideweg zwischen Musik und Malerei, oder mehrere Bilder mit musikalischen Museum.

${ }^{17}$ In Schwarzenberg, der „Wahlheimat“ der Malerin, befindet sich das Angelika Kauffmann

18 So schreibt über Kauffmann ihr erster Biograph Rossi, Eduard Engels betitelt als Wunderkind ein Kapitel seiner Biographie.

${ }^{19}$ Schon damals machte sie auf Wunsch ihres Vaters mehrere Sitzungen ,im Grünen Zimmer“, arbeitete viel und malte Porträts von reichen Adeligen.

${ }^{20}$ Vgl. auch ebd., S. 59.

21 Durch diese Stilisierung zur Sängerin wertete sich junge Malerin auf, denn musikalische sowie Gesangsausbildung gehörte zu Privilegien adeliger Töchter (s. Katz 2012: 9). 
Motiven und Attributen (Gemälde Drei Sängerinnen, Porträts mit einem Notenblatt oder Musikinstrument). ${ }^{22}$ Kauffmann spielte Cembalo ${ }^{23}$ und Zither; sie liebte Musik so sehr, dass sie gern nicht nur ihren Gästen im Salon vorsang (z.B. Pergolesis Salve Regina - Blick 125, 133), sondern auch während der Sitzungen zu Porträts im Atelier.

Entzückt von Angelikas künstlerischem Talent ist der amerikanische Arzt, Wissenschaftler und Kunstkenner John Morgan, den Kauffmann in Rom porträtierte (Blick 94-95). Mit Bewunderung äußert er sich über Angelikas gute Kenntnis des menschlichen Körpers (vgl. Blick 95), obwohl sie als Frau keine männlichen Akte zeichnen konnte und die männliche Anatomie aus der Beobachtung antiker Skulpturen erlernen musste. Ebenso durfte sie keine Akte malen, deswegen sind ihre Figuren meistens gekleidet oder tragen eine Toga. In der patriarchalischen, von den Männern dominierten Welt betrachtete man ,[...] den weiblichen Körper als Abweichung vom männlichen [... ]“" (Blick 95). Die erste Römer Periode bringt ihr wichtige Bekanntschaften mit den Malern Benjamin West und Nathaniel Dance und vornehmlich mit dem Altertumsforscher und Kunstkenner Winckelmann, den sie porträtiert und dessen Ideen ausschlaggebend für ihre weitere künstlerische Laufbahn werden. Winckelmann wird für Kauffmann zur Leitfigur, sein Idealismus und Auffassungen über die Schönheit, Grazie (vgl. Blick 84) und Harmonie in der Kunst finden ihren Niederschlag im Euvre der Malerin.

Eine etwas kritische Attitüde zu Kauffmann vertritt der Schweizer Maler und Dichter Johann Heinrich Füssli, der ebenfalls in London wirkt und Angelika kennt. Er erzählt zuweilen etwas spöttisch über ihre Verehrer ${ }^{24}$ (Benjamin West und Nathaniel Dance, möglicherweise auch Sir Joshua Reynolds) und Förderer (Reynolds, Leiter der Royal Academy, der auch Füssli unterstützte). Kauffmann wird in London dank ihrem Porträt des Schauspielers und Dichters David Garrick (Blick 117) bekannt, der ebenfalls in sie verliebt sein sollte. Das Bildnis wird in London auf einer Ausstellung präsentiert, noch bevor Kauffmann nach England kommt und ihr eigenes Studio eröffnet. Es gehört bald zum guten Ton, sich von der Malerin porträtieren zu lassen: Sie malt die Reichsten vom Hof und Adel, aber auch Künstler, Musiker, Dichter und Gelehrte. Vom großen Erfolg der Schweizer Malerin zeugt die Tatsache, dass sie eines der Gründungsmitglieder der renommierten Royal Academy of Arts ist. Sie wird auch auf dem Gemälde der Königlichen Akademie von Johann Zoffany (1771/72) - neben der Stilllebenmalerin

22 Angelikas Mutter Cleophea wollte, dass sie Sängerin wird und gab ihr Musik- und Gesangsunterricht. Zur Musik im Schaffen von Kauffmann s. Petra Zudrell / Reinhard Strohm / Tobias Bonz / Barra Boydell: Zwischen Musik und Malerei. Gestaltung: Frank Broger. Katalog, Angelika Kauffmann Museum, Schwarzenberg 2012.

${ }^{23}$ Auf dem Familienbildnis des Grafen Ely ist u.a. Angelicas Cembalo zu sehen, das sie nach Irland mitnahm (s. Blick 226).

${ }^{24}$ Nach manchen Überlieferungen sollte Füssli selbst in Kauffmann verliebt sein, dann in Mary Moser, bis er schließlich eine andere Frau heiratete - vgl. Blick, S. 222-223. 
Mary Moser ${ }^{25}$ - berücksichtigt. Da Zoffany unter den angesehenen Professoren und Malern zwei nackte, posierende Männermodelle darstellte, was für die Damen damals eine unerhörte Gesellschaft bedeutete, wurden beide Malerinnen taktvoll in Form von Porträts an der Wand rechts platziert.

Alioth zeigt nicht nur Erfolge (vgl. Blick 35), sondern auch Tiefpunkte im Leben der Malerin: Kauffmanns Errungenschaften wecken einerseits Bewunderung, andererseits Neid und Eifersucht der Malerkollegen in London und Rom (vgl. Engels 1903: 109), weswegen es zu Skandalen kommt. Viele Hypothesen und Mutmaßungen weckt die erste Ehe Kauffmanns mit dem falschen, schwedischen Grafen de Horn, den sie im Herbst 1867 heiratet und der sich als Heiratsschwindler entpuppt (vgl. auch Blick 218-224). Angelikas Vater versucht die Mesalliance zu vertuschen und die Ehe wird nach drei Monaten für ungültig erklärt. Wegen dieser gescheiterten Liebe, die für die junge Malerin wohl eine große persönliche Tragödie bedeutet, leidet Kauffmann auch noch lange Zeit danach, wovon die Szene mit Herder zeugt (Blick 186). Ein anderer Skandal bricht während der Ausstellung des Gemäldes The Conjurer (1775, Der Zauberer ${ }^{26}$ vom enttäuschten und eifersüchtigen irischen Maler Nathaniel Hone aus, der auf seinem Bild Sir Joshua Reynolds und die nackte Angelika darstellt. Auf diese Weise rächt er sich an den Mitgliedern der Royal Academy, weil er sich an der Bemalung der Kirche St. Paul nicht beteiligen durfte (Blick 123).

Auch der mit Angelika Kauffmann befreundete Kunsthändler Reiffenstein ${ }^{27}$ ist von ihrer Kunst begeistert: Er widmet dem längeren Aufenthalt von Goethe in Italien und Kauffmanns großer Faszination des Weimarer Dichters viel Aufmerksamkeit. Er suggeriert sogar Angelikas Liebe zu Goethe, ${ }^{28}$ obwohl sich die vom Vater arrangierte zweite Ehe mit dem venezianischen Vedutenmaler Antonio Zucchi $^{29}$ als glücklich erweist. Die Malerin wird von der Zucchi-Malerfamilie gut aufgenommen und zwar so sehr, dass Angelikas Schwager Carlo Zucchi Materialien und Informationen über sie sammelt (Blick 197), ${ }^{30}$ die später von ihren Biographen verwendet werden. Zuletzt besucht der Ich-Erzähler Carlo Zucchi in Venedig, wo sich herausstellt, dass er der Halbbruder von Angelika ist. Diese Wahrheit hat er zwar von seinem Vater Johann Kauffmann nach dem Tode seiner

${ }^{25}$ Mary Moser war die Tochter eines Schweizer Goldschmieds Georg Moser. Der brit. Maler Richard Samuel porträtierte Kauffmann als eine der Musen (sie sitzt vor der Staffelei) auf seinem Gemälde der Neun Musen des Tempels von Apollo (1777).

26 S. Blick, S. 119-120, 223.

${ }^{27}$ Hofrat Johann Friedrich Reiffenstein (1719-1793) war ein in Rom sesshafter Deutscher, der in der Nähe von Kauffmann wohnte.

${ }^{28}$ Eine ähnliche Hypothese entwickelt auch Ursula Naumann im Buch Geträumtes Glück. Angelica Kauffmann und Goethe. S. auch Irmgard Smith-Dörrenberg: Angelika Kauffmann. Goethes Freundin in Rom. Wien 1968.

${ }^{29}$ Zucchi (1726-1795) war Sohn des Malers Francesco Zucchi in Venedig. Es gibt Vermutungen, dass diese Ehe nie konsumiert wurde (Goodden 2005).

${ }^{30}$ S. Anm. 7. 
Mutter erfahren, er hat sie aber nie geglaubt und verdrängt, weil er ein uneheliches Kind war und seinem Vater Diskretion versprach. Nach anderen Überlieferungen soll Franz Joseph Kauffmann aus der ersten Ehe von Johann Kauffmann ${ }^{31}$ stammen. In etwas ungünstigem Licht zeigt Alioth Angelikas „Seelenfreund“32 Herder, der in Der prüfende Blick nicht nur zu den größten Verehrern der Malkunst von Kauffmann gehört, sondern in sie verliebt ist.

Die Schweizer Schriftstellerin präsentiert ein differenziertes, widersprüchliches (Skandale), im allgemeinen aber sehr positives Bild von Kauffmann, die trotz der Erfolge immer dieselbe bescheidene Person blieb, was ihre Zeitzeugen zu schätzen wissen: Der Priester von Schwarzenberg erinnert sich nach Jahren an ihre Tugendhaftigkeit und ihren tiefen Glauben an Gott, um den er sie fast beneidet. Der Sänger von Tettnang schildert sie als anmutig, zart (Blick 68, 87) und mit guten Manieren, was damals im gesellschaftlichen Leben eine große Rolle spielte. Der Musiker lobt auch ihr Talent für Fremdsprachen (Kauffmann sprach Deutsch, Englisch, Italienisch, Französisch), ihre Lernfähigkeiten, sowie ihr Interesse für Legenden und antike Sagen. John Morgan ist entzückt von ihrem persönlichen Charme, Takt und Anmut der „zarten Person“ (Blick 87). In höchsten Tönen preist sie Herder und bezeichnet sie als „eine liebe Seele“, „einen Engel mit einem Pinsel“ (Blick 177), ,jungfräuliche Engelsseele“ (Blick 183), „die kultivierteste Frau Europas“ (Blick 182), oder lobt ihre Empathie: „die Muse der Menschlichkeit sei ihre Schwester“ (Blick 187). ${ }^{33}$ Eine kritische Stimme stammt von Füssli, der - neidisch auf ihren kommerziellen Erfolg - unterstellt der Malerin finanzielle Motive. Nach London sei sie angeblich übergesiedelt, nicht „um zu malen, sondern um Geld zu verdienen [...]“ (Blick 122). ${ }^{34}$ Reiffenstein kritisiert Angelikas übermäßige Gutmütigkeit und allzu große Passivität sowie Geduld gegenüber anderen Menschen, denen sie sich nicht zu widersetzen vermag (Blick 191), während der Ich-Erzähler konstatiert, dass sie allen gefallen wollte. ${ }^{35}$ Nach Carlo Zucchi sollte sie Füssli ausgenutzt haben, um an höhere Kreise in London zu kommen (Blick 223).

Gabrielle Alioth zeigt Angelika Kauffmann als eine vielseitig talentierte, sensible und bescheidene, zugleich aber auch ungewöhnlich starke und selbstbewusste Persönlichkeit, der es gelang, sich in ihrer frauenfeindlichen Epoche

${ }^{31}$ Nach Alioth ist der Ich-Erzähler das uneheliche Kind von Johann Kauffmann. Nach Jutta Rebmann war Joseph Kauffmann (geb. am 10. März 1734 in Herrenalb) Angelikas Halbbruder aus der 1. Ehe ihres Vaters mit Maria Sybilla Lohrin (s. auch Katz 2012: 12).

32 So bezeichnet Herder Engels in seiner Biographie und schildert Herders Verhältnis mit Kauffmann als rein freundschaftlich.

${ }^{33}$ In seiner Korrespondenz bezeichnete Herder Kauffmann als „eine Perle“ (zit. nach Engels 1903: 131) oder schrieb über sie: ,die wahre, himmlische Muse voll Grazie, Feinheit, Bescheidenheit und einer ganz und unnennbaren Güte des Herzens“ (ebd., S. 132).

34 Vgl. auch: ,Jede Geschichte, die sich verkaufen ließ, nahm sie auf“" (Blick 125).

35 Alioth selbst sprach von der „,angepassten, gefälligen Angelica“ (Alioth 2015: 16). 
als freie Künstlerin durchzusetzen. ${ }^{36}$ In gewissem Sinne realisierte sie Schillers Ideal der „schönen Seele“, worauf Klaus Hammer (s. Hammer 2007) mit Recht verweist. Obwohl sie keine Schönheit war, sollten mehrere Männer in sie verliebt gewesen sein. Ihr ganzes Leben lang musste sie hart arbeiten, um den Lebensunterhalt für ihren Vater (s. Blick 87), dann für ihren zweiten Mann zu verdienen. Sie ließ sich nicht als Hofmalerin engagieren (vgl. Blick 212), sondern eröffnete eigene Ateliers in London und Rom, was ihr künstlerische Freiheit sicherte. Was selten der Fall ist, genoss sie seit früher Jugend Erfolg und Anerkennung. ${ }^{37}$ Ihre Kunst - verbreitet durch Kupferstiche, Radierungen und Porzellanbilder - erfreute sich solchen internationalen Ruhms und solcher Popularität, dass sie mehr Aufträge bekam, als sie annehmen und ausführen konnte (vgl. Maierhofer 1997: 113). Ihr Genie, Talent und ihre einzigartige Persönlichkeit besangen Dichter (Fr. v. Matthisson, Graf zu Stolberg, George Keate), lobten Künstler, Schriftsteller (Goethe, Klopstock, Herder) und wichtige Persönlichkeiten der Epoche. Sie fertigte Zeichnungen zu Shakespeare, Klopstocks Messias, Goethes Egmont und Iphigenie auf Tauris oder Wielands Oberon an. Angelika Kauffmann führte in Rom einen bekannten künstlerischen Salon, in dem sich viele Persönlichkeiten der geistigen Elite und Aristokratie sammelten, hatte eine eigene ,prächtige Kutsche“ (Blick 212), galt als einflussreiche Person und wurde von zahlreichen Künstlern aus Deutschland besucht (Goethe, Herder, Dalberg). Was für ihre Epoche kennzeichnend ist, legte sie großen Wert auf Freundschaften ${ }^{38}$ und gute Kontakte mit vielen Persönlichkeiten, schrieb Briefe in vier Sprachen ${ }^{39}$. Zu ihren Freunden gehörten Joshua Reynolds, Benjamin West, Reiffenstein, Goethe, Herder, der Bildhauer Antonio Canova oder Herzogin Amalia.

Im Gegensatz zu Angelika wird ihr Vater Johann Kauffmann an mehreren Stellen im Roman von einigen Figuren sehr kritisch als „engherzig“ und „knauserig“, „habgierig“, ,abweisend“, oft „übellaunig“ (s. Blick, entsprechend 33, 61, $65,89,176)$ beurteilt. Er sollte Angelika das Singen während der Arbeit verbieten (Blick 51) oder sie in einem unsichtbaren „Netz" halten (Blick 221) und für eigene Zwecke ausnutzen (Blick 42). Auch der Landschaftsmaler erinnert sich eher ungern an den alten Lehrmeister, anders als an Angelikas Mutter Cleophea, die ihn

${ }^{36}$ S. dazu: dieses Mädchen hatte ,[...] immer nur eines gewollt [...]: Malen und Zeichnen, dazu Singen und Musizieren“ (Rebmann 2007: 10).

$37 \mathrm{Zu}$ ihrem Erfolg und Rezeption um 1800 s. bei früheren Autoren: Düringsfeld 1906: 143; Engels 1903: 162.

38 S. dazu Wolfram Mauser / Barbara Becker-Cantarino (Hrsg.): Frauenfreundschaft, Männerfreundschaft: Literarische Diskurse im 18. Jahrhundert. Tübingen 1991.

39 Sie korrespondierte u.a. mit Klopstock, J.C. Lavater, S. Gessner, Goethe oder Herder. Einen Teil ihrer Korrespondenz hat Kauffmann vor ihrem Tode verbrannt. Ihre Briefe veröffentlichte Waltraud Maierhofer (Auswahl, Kommentar u. Einl.): Angelika Kauffmann - Briefe einer Malerin. Mainz 1999. Siehe auch Waltraud Maierhofer (Hrsg.): Angelika Kauffmann. „Mir träumte vor ein paar Nächten, ich hätte Briefe von Ihnen empfangen “. Gesammelte Briefe in den Orginalsprachen. Lengwil 2001. 
herzlich behandelte. Johann Kauffmann ${ }^{40}$ sollte Angelika zu viel Arbeit gedrängt haben und war „stets im Studio anwesend [...]“ (Blick 86). Vergessen wird dabei die Tatsache, dass Kauffmann in gewissem Sinne die Karriere der Tochter ermöglichte, leitete und sie als Mann beschützte - in der damaligen Epoche konnte sie als Frau allein nicht funktionieren. ${ }^{41}$ Trotz seines dominanten und schwierigen Charakters konnte das malerische Talent Angelikas unter seiner Betreuung erblühen. Der Vater empfing die Aufträge, übernahm die Buchführung und erfüllte die Rolle eines Managers oder Kunstagenten. Nach dem Tode des Vaters übernahm diese Aufgabe zunächst Antonio Zucchi, später Angelikas Vetter aus Deutschland, der auch Johann Kauffmann hieß.

Alioths mosaikartiges, literarisches Porträt der Künstlerin besteht aus vielen Berichten, Meinungen und Urteilen ihrer Zeitgenossen über die Künstlerin und ihr Werk. In ihrem Roman Der prüfende Blick liefert die Schriftstellerin auch zahlreiche Ekphrasen (Bildbeschreibungen) ${ }^{42}$ und schildert mehrere Sitzungen zu Porträts, wobei sie sich insbesondere auf Bildnisse konzentriert, etwas weniger Aufmerksamkeit den bekannten Historienbildern widmet. Ihre Sprachfähigkeiten, die große Anpassungs- und Kontaktfähigkeit, intuitive Menschenkenntnis ${ }^{43}$ sowie scharfes Beobachtungsvermögen (ihr ,prüfender Blick“-Blick 38, 44, 86, 170, 234) ermöglichten der Malerin, sich in die Seele und den Charakter der abgebildeten Personen einzufühlen (s. Blick 149, 234). Während ihrer Sitzungen, die oft ein ästhetisches Erlebnis waren, wurden Gedichte oder Dramen vorgelesen (z.B. Goethes Iphigenie), Gespräche über Literatur, Kunst und Musik geführt. Dank ihrer Intelligenz, großer Belesenheit sowie solider Orientierung in den Kunstfragen (sie kannte alle bekanntesten Kunstwerke sowie Kunsttheorien und -auffassungen), ${ }^{44}$ war sie eine ebenbürtige Gesprächspartnerin für Goethe oder Herder.

Der zweite Pfad zu Angelika Kauffmann führt bei Gabrielle Alioth über ihre Malerei, die im ganzen Roman ständig präsent ist - in Form von zahlreichen Bildbeschreibungen, Interpretationen oder Erwähnungen. Dadurch kommt indirekt die Malerin selbst zu Wort, die mittels ihrer Werke mit dem Leser kommuniziert. Den

${ }^{40}$ Kauffmanns Bildnis des Vaters aus Italien zeigt ihn als eine starke Persönlichkeit (um 1761-4).

${ }^{41} \mathrm{Zu}$ erinnern sei hier an die Tatsache, dass man damals die Frauen als weniger intelligent betrachtete, den Männern unterlegen, sie sollten sich allein der Familie widmen. Ihr musikalisches Talent konnten damals u.a. die Schwester von Mozart oder Goethe nicht entwickeln. Schiller musste die Bücher für seine Frau und Schwägerin ausleihen, weil es den Frauen nicht erlaubt war. Vgl:: „Aber das Genie der Frau ist so flüchtig, dass die Umstände ihm nur selten erlauben, sich zu entfalten“ (Blick 123). Nach Goethe hatte Kauffmann „für eine Frau ein wirklich ungeheures Talent“ (Blick 156).

42 Ekphrasis, d.h. die Beschreibung eines Kunstwerkes, wurde zuerst in Reiseberichten (Lessing, Goethe) verwendet, um sie als ästhetisches Erlebnis darzustellen. Zum Begriff s. Roma Sendyka: Esej i ekfraza (Herbert-Bieńkowska-Bieńczyk). In: Przestrzenie Teorii Nr. 11, S. 41-61. Alioth beschreibt auch Bilder anderer Maler, wie J.H. Füssli, N. Hone, N. Dance oder H.G. Schütz.

${ }^{43}$ Nach Reiffenstein hat sie sich nie ,von Rang und Namen blenden lassen“ (Blick 149).

44 Vgl. Goethes Worte über Kauffmann: ,[...] Ihr Auge sei gebildet, ihre Kunstkenntnisse seien groß und ihr Sinn für das Schöne und Zarte so ausgeprägt“ (Blick 159). 
Charakter ihrer Kunst, ihren Stil, aber auch Thematik und formelle Fragen (Form, Farben und Komposition $)^{45}$ prägten die Wegbereiter und Theoretiker des Klassizismus, der Maler Anton Raphael Mengs (siehe Blick 120, 152) und vornehmlich der Kunsthistoriker Winckelmann, der im Roman mehrmals erwähnt wird (Blick 94, 95, 97-98). Schon in ihrer Jugend wurde Kauffmann mit den damals neuesten und fortschrittlichsten Ideen der Epoche konfrontiert. Als die Malerin im Alter von 23 Jahren Winckelmann in Rom malte (1764), war sie eine Anfängerin, die noch alte Meister kopierte (Raffael, Rembrandt, Correggio) und auf der Suche nach einem eigenen Stil war, während Winckelmann den Ruf eines bedeutenden Gelehrten genoss. Nach Engels hat Winckelmann sie „von oben herab“ behandelt (Engels 1903: 54), obwohl ihm Kauffmanns Arbeit gefiel. Die junge Malerin betrachtete die Ideen von Winckelmann und Mengs als Richtlinien für ihren Weg als Künstlerin: In ihren Veröffentlichungen Mengs in Gedanken über die Schönheit und über den Geschmack in der Malerey (Blick 92, 132), Winckelmann in Geschichte der Kunst des Altertums (Blick 97) formulierten sie die Aufgaben der Kunst: „Die Darstellung des Schönen [...] müsse das höchste Ziel des Künstlers sei“" (Winckelmann - Blick 93). Ähnliche Anschauungen vertrat Mengs, dessen Ideen Kauffmann vom Maler Benjamin West kannte:

Schönheit [...] [sei] eine Erinnerung an die göttliche Vollkommenheit und deshalb in allen Dingen zu finden [...]. Denn die Natur hat nichts Unnützes geschaffen [...]. Und wenn die Kunst der Natur in der Nachahmung von Licht und Finsternis auch stets unterlegen sei, so könnte sie [...] aus dem Erschaffenen das Schönste auswählen und sie - durch Auslassung alles Unbedeutenden - übertreffen... (Blick 92)

Kauffmann war besonders beeindruckt von Winckelmanns Ansichten über die Einheit von Schönheit und Wahrheit, von der Grazie und Anmut (Von der Grazie in Werken der Kunst), sowie von seiner Idee der Fähigkeit mancher Menschen, „das Schöne in der Kunst zu empfinden“(Winckelmann 1982: 138). Diese „himmlische“ Gabe „der Empfindung des Schönen“ (ebd.) erfordert von Künstlern und Kunstrezipienten besondere Seelenqualitäten, die durch entsprechende Bildung geweckt und gestaltet werden können.

Gemäß den Hinweisen von Winckelmann besuchte Kauffmann alle für die Künstler bedeutenden Orte in Italien, lernte alle wichtigsten Kunstwerke und Herkulaneum kennen, beteiligte sich ebenfalls an Kunstausstellungen. Inspiration für ihre Werke suchte sie in der antiken Kunst und Literatur (Homer, Vergil, Sappho), in Sagen und der Mythologie, aber auch bei Klassikern wie Shakespeare und Tasso, oder zeitgenössischen Dichtern wie Klopstock, Gellert oder Goethe. Entgegen der damals herrschenden Verhältnisse hat sie sich in den „höchsten“ Formen der „männlichen“ Malerei ${ }^{46}$ wie der Historienmalerei etabliert, sie griff zuweilen auch

45 Vgl. hierzu Winckelmanns Abhandlung von der Fähigkeit der Empfindung des Schönen in der Kunst und dem Unterrichte derselben (1763). In: Winckelmann 1982: 158-159.

${ }^{46}$ Nach der damals fungierenden Rangordnung in der Malerei galt das weniger bedeutende Stillleben als für die Frauen „entsprechend“, die Landschaftsmalerei befand sich auf der niedrigsten Stufe. Erst mit C.D. Friedrich gewann die Landschaftsmalerei an Bedeutung. 
religiöse Themen auf. Dank ihrem Talent, ihrer Offenheit, Lernbereitschaft aber auch vielen Lektüren und unermüdlicher Arbeit hat sie ihren eigenen, unverkennbaren Stil gefunden ${ }^{47}$ Ihre Historienbilder sind sofort erkennbar durch originelle Themen, charakteristische Figuren und ihre dynamischen Posen. Sie spezialisierte sich auf stilisierte Selbstbildnisse und allegorische Rollenporträts mit mythologischen oder literarischen Konnotationen.

Alioths Roman enthält mehrere Beschreibungen von Kauffmanns Selbstbildnissen, die die vielseitige Kunstschaffende in verschiedenen Phasen ihrer künstlerischen Laufbahn zeigen: Ihr erstes Selbstbildnis im Rokokokleid, das ihre Sängerambitionen verrät, das Selbstporträt in Bregenzerwälder Tracht (Blick 35), womit Kauffmann ihre Wahlheimat Schwarzenberg würdigt; das Selbstbildnis im weißen Kleid aus der Galerie der Uffizien in Florenz ${ }^{48}$ (1787 - Blick 174), das ihren Ruhm und hohen künstlerischen Rang betont, oder das wohl berühmteste Gemälde Selbstbildnis am Scheideweg zwischen Musik und Malerei ${ }^{49}$ (1792 - Blick 190), das ihre inneren Kämpfe wegen ihrer Doppelbegabung und die schwierige Entscheidung über den richtigen Lebensweg allegorisch darstellen. Auf Selbstbildnissen ist Kauffmann in verschiedenen Posen meistens als Malerin mit Pinsel und Mappe zu sehen: Die Malerattribute und schöne, teure Kleider nach neuester Mode versinnbildlichen ihren hohen beruflichen und gesellschaftlichen Status, zugleich zeigen die Porträts sie als subtile, zarte, empfindsame und verständnisvolle Frau. Kauffmann malte befreundete Persönlichkeiten (Goethe, Herder, Sir Joshua Reynolds), sie wurde aber auch von anderen gemalt, wie Reynolds, Nathaniel Dance (Blick 92-93) oder Benjamin West (Blick 92). Mit anderen Ekphrasen verweist Alioth entweder auf wichtige Kunstwerke der Malerin (Porträts von Winckelmann, Garrick, Porträt der Herzogin Amalia von Sachsen-Weimar-Eisenach mit der Büste von Minerva und Herder-Buch, Zyklus der Vier Elemente der Malerei für die Londoner Ausstellung) oder auf Aufträge, die ihre Anerkennung und ihr hohes Ansehen als Künstlerin in der damaligen Welt dokumentieren: Das Familienbildnis des Vizekönigs Townshend von Irland (Blick 225), der Familie des Grafen Ely in Irland (mit dem schwarzen Diener und Cembalo - Blick 226), das Bildnis des polnischen Prinzen Stanislaw Poniatowski (Blick 146), oder das großformatige Familienporträt des Ferdinand IV., Königs von Neapel und Sizilien (Blick 152-153). Kauffmann malte Könige und die reichsten Vertreter der europäischen Aristokratie, ihre Bilder waren weit verbreitet und erreichten hohe Preise: „Für den Preis eines ihrer Bilder hätte sie das halbe Dorf kaufen können“ (Blick 35).

${ }^{47}$ Ihr Freund Reiffenstein konstatiert: „So begierig war sie, von allen zu lernen, dabei trug sie das Wichtigste in sich“(Blick 149). Vgl. auch Maierhofer 1997: 73.

48 Vgl. hierzu Maierhofer 1997: 113 oder Düringsfeld 1906: 159.

49 Somit knüpft Kauffmann an das bekannte Renaissancegemälde von Anniballe Carracci Herkules am Scheideweg (1596) an. Dieser Verweis auf eine berühmte antike Figur belegt ein hohes Selbstwertgefühl der Malerin. Vgl. auch Lettau 1998. Über Carracci schreibt Winckelmann in seiner Abhandlung von der Fähigkeit der Empfindung des Schönen. 
Gabrielle Alioth interessieren bei Kauffmann insbesondere Gefühle und das Motiv der Liebe (Liebes- und Trennungsschmerz, Sehnsucht, Enttäuschung, Verzweiflung, Traurigkeit, Leiden, Trauer) und sie verweist mit Recht darauf, dass Kauffmann für ihre Malerei nicht heroische Momente wählt, sondern emotional bewegende Szenen aus der Mythologie und Literatur, wie Hectors Abschied von Andromache, Der trauernde Telemach auf der Insel der Kalypso oder Die irre Marie. Alioth versucht auch, die Hintergründe der Malerei zu erforschen und die Gefühls- und Gedankenwelt der Malerin nachzuvollziehen. In Orpheus und Eurydice (Blick 69-72) oder Kauffmanns Lieblingstopos der verlassenen Ariadne und Bacchus (Blick 88) sieht die Schriftstellerin die Wiederspiegelung der Gemütszustände der Malerin. Möglicherweise beeinflusste der Liebeskummer nach der Trennung von einem Sänger ihre Entscheidung für die Malerei (vgl. Blick 72). Nach Reiffenstein entstand Vergil liest aus der Äneis für Oktavia ${ }^{50}$ (Blick 173) als Ausdruck von Kauffmanns Sehnsucht nach Goethe. Mit Psyche und Amor würdigt die Malerin die Reinheit der Liebe (Blick 173-174). Manchmal wählt Kauffmann für ihre Allegorien Szenen aus der Mythologie oder Literatur, die moralisch-ästhetische Funktion ${ }^{51}$ erfüllen: Die Penelope-Bilder versinnbildlichen die Tugend der Ehetreue, Cornelia, die Mutter der Gracchen zeigt das Ideal der echten Mutter, die ihre Kinder als wahren Schatz empfindet (Blick 161), nicht die teuren Schmucksachen. An vielen Gemälden ist Kauffmanns Liebe zur Kunst, Musik und Literatur (Mythen) abzulesen, wie beispielsweise Bacchus lehrt die Nymphen die Dichtkunst (Blick 146-147) oder Die Dichtung umarmt die Malerei. Die Büsten von Minerva, Michelangelo oder Homer, oder Reliefs mit drei Grazien auf den Porträts betonen zusätzlich die Vorrangstellung der Kunst und Literatur im Leben der Malerin. Popularität brachten Kauffmann auch leichtere, humorvolle Szenen (z.B. mit dem Amor oder tanzenden Grazien), was Füssli abwertend als Kommerzialität bezeichnete. Es gibt auch gemeinsame Arbeiten der Malerin mit anderen Künstlern, wie beispielsweise das Gemälde Nymphen löschen das Feuer der Liebe mit Philipp Hackert.

Ihr Talent - so Alioth - Arbeit, Originalität aber auch Sanftmut (Senn-Schnitzler 1952: 27), persönlicher Charme, Takt und diplomatische Umgangsformen, die ihr gute Kontakte mit Menschen erleichterten, trugen wohl zum Erfolg von Angelika Kauffmann bei, die als erste professionelle, freiberufliche, deutschsprachige Künstlerin in Europa wirken konnte. ${ }^{52}$ Sie hatte den Mut und die Kraft, der inneren Berufung und der Liebe zur Malerei, ${ }^{53}$ aber auch der Musik und Literatur zu folgen. Kauffmann war allgemein beliebt, man sprach über die unkonventionelle Frau mit

${ }^{50}$ Dieses Bild entstand auf Bestellung von Stanisław Poniatowski von 1787.

51 S. dazu Marie-Louise Geiseler: Die Schönheit am Scheideweg. Schönheit und Mäßigung in den Tugend- und Kunst-Allegorien von Angelika Kauffmann. In: Zeitschrift für Kunstgeschichte, 1.01.2004, Bd. 67(1), S. 131-141.

52 Anna Dorothea Therbusch-Lisiewska war zwar eine Generation älter, wirkte früher, war aber nicht so bekannt wie Kauffmann und arbeitete als Hofmalerin.

${ }^{53}$ Vgl. dazu: Kauffmann war „,vom großen Ziel beseelt“, eine gute Malerin zu werden (Blick 87). 
Sympathie als „Miss Angel“, bezeichnete sie als „,die zehnte Muse von Rom“, „Malerin der Grazien“ (Wieland - Blick 191) oder war „,verrückt nach Angelika“ (Klopstock). Sie verwirklichte Schillers Konzeption der „schönen Seele“, noch bevor sie formuliert wurde ${ }^{54}$ Alioth betont die besondere Gabe der Malerin, das Schönste in den Menschen hervorzuheben, sie „vollkommener zu malen“ (vgl. Blick 149), sowie ihre Fähigkeit, ein Stück von sich selbst den abgebildeten Personen zu geben. ${ }^{55}$ In Kauffmanns Welt sollen Kunst, Musik und Literatur das menschliche Leben bereichern und veredeln. Liebe, Grazie und Harmonie sind ständige Elemente ihrer „weiblichen“, „zärtlich schwärmenden“ (Engels 1903: 144) Malerei, die auf einzigartige Weise Schönheit und Empfindsamkeit vereint. Intelligente und schöne Kunst von Kauffmann wirkte des Öfteren sensibilisierend und erhebend, aber regte auch mit vielen ästhetischen Assoziationen und literarisch-mythologischen Verweisen zur Beschäftigung mit Literatur, Musik und Kunst an, ${ }^{56}$ trug dadurch - wenn auch indirekt - zur Verbreitung der positiven Mode auf Bildung bei. Kauffmanns Kunst wird manchmal als allzu sentimental oder zu weich (z.B. androgyn wirkende, sublimierte Männerfiguren anstatt von starken Heroen ${ }^{57}$ ) abgewertet und als veraltet kritisiert. ${ }^{58}$ Ein neues, wachsendes Interesse am „artistic phenomenon“ (Goodden 2005) Angelika Kauffmann (neue Biographien, erfolgreiche Ausstellungen) in den letzten Jahren belegt, dass sie auch den heutigen Rezipienten ansprechen und berühren kann. Anders als andere Autoren konzentriert sich Gabrielle Alioth auf die Persönlichkeit und das Werk der Malerin, nicht auf sensationelle Seiten ihrer Biographie (vgl. Klüger 2010: 151). Alioths vielfältiges, intermediales, literarisch-malerisches Porträt erinnert an die talentierte und sensible Malerin, ihre ungewöhnliche Biographie und lädt zur Auseinandersetzung mit ihrer originellen Malerei ein.

\section{Literatur}

Alioth, Gabrielle (2007): Der prüfende Blick. Roman über Angelica Kauffmann. München.

Alioth, Gabrielle (2015): Historische Erfindung oder erfundene Historie? In: Pender, Malcolm / Burns, Barbara (Hrsg.): Konstruktionen der Vergangenheit in der Deutschschweizer Literatur. Würzburg. S. 9-18.

${ }^{54}$ Auf das Ideal der ,schönen Seele“ geht Alioth im Roman expressis verbis nicht ein (s. Hammer 2007), sie tut es aber in Alioth 2015: 11. Kaufmanns Biograph lobte vor über 100 Jahren ihre „holde Seelenanmut“ (Engels 1903: 144).

55 Gemeint ist eine gewisse Ähnlichkeit der Abgebildeten mit der Malerin.

56 Der Ich-Erzähler bekennt, dass er die Sagen und Mythen, an die Angelika anknüpft, nicht kennt (Blick 100).

${ }^{57}$ Eben deswegen missfiel Goethe sein Porträt von Kauffmann, weil sie ihn als sensiblen, empfindlichen Künstler darstellte.

58 Vgl. z.B.: ,Ihre delikate und sentimentbehaftete Malerei ist selbst noch im klassischen Aufbau ein graziöses Relikt aus dem Zeitalter der Empfindsamkeit““(Lettau 1998). 
Alioth, Gabrielle (2007): Schreibinseln. Betrachtungen zum Lesen und Schreiben zwischen der Schweiz und Irland. In: Studer, Patrick / Egger, Sabine (Hrsg.): From the Margins to the Centre. Irish Perspectives on Swiss Culture and Literature. Bern. S. 51-60.

Bachmann, Eva: Ähnlich, doch vollkommener. In: St. Galler Tagblatt 26.02.2007.

Baumgärtel, Bettina (1990): Angelika Kauffmann (1741-1807). Bedingungen weiblicher Kreativität in der Malerei des 18. Jahrhunderts. Diss., Weinheim.

Baumgärtel, Bettina (1995): „Der weibliche Raphael der Kunst“: Werke der Angelika Kauffmann in Schweizer Sammlungen. Stämpli.

Birrer, Sibylle: Sittenbild, mit Dame im Hintergrund. Gabrielle Alioths Roman über Angelica Kauffmann. In: Neue Zürcher Zeitung 9.05.2007.

Borzello, Frances (2012.): Sobald ich vor der Leinwand saß. Künstlerinnen aus fünf Jahrhunderten. Üb. aus Engl. C. Panzacchi. Hildesheim.

Borzello, Frances (1998): Wie Frauen sich sehen. Selbstbildnisse aus fünf Jahrhunderten. Üb. aus Engl. K. Tschumper. München.

Donovan, Siobhán (2008): Der romantische Sog des Wassers, des weiblichen Elements. Zu Gabrielle Alioths Roman „Die Arche der Frauen“. In: Goodbody, Axel / Wanning, Berbeli (Hrsg.): Wasser - Kultur - Ökologie. Beiträge zum Wandel im Umgang mit dem Wasser und zu seiner literarischen Imagination. Göttingen. S. 285-300.

Düringsfeld, Ida von (1906): Angelika Kauffmann. In: Dies.: Das Buch denkwürdiger Frauen. Lebensbilder und Zeitschilderungen. 7. Aufl. Leipzig. S. 143-160.

Engels, Eduard (1903): Angelika Kauffmann. Reihe: Frauenleben, hrsg. v. Hanns v. Zobeltitz. Bielefeld/Leipzig.

Fliedl, Konstanze / Oberreither, Bernhard / Serles, Katharina (Hrsg.) (2013): Gemälderedereien. Zur literarischen Diskursivierung von Bildern. Berlin.

Gerard, Frances A. (1893): Angelica Kauffmann. A Biography. [Mit Auflistung der Werke]. New York.

Goodden, Angelica (2005): Miss Angel. The Art and World of Angelica Kaufman, Eighteenth-Century Icon. London.

Hammer, Klaus: Auf der Spur einer Suchfigur. Gabrielle Alioth hat einen etwas verwirrenden Roman über die Malerin Angelika Kauffmann geschrieben. In: literaturkritik.de 29.08.2007, http://literaturkritik.de/public/rezension.php?rez_id=11103\&ausgabe=200709 (Zugriff am 7.04.2018).

Helbok, Claudia (1968): Miss Angel. Angelika Kauffmann - eine Biographie. Wien.

Herder, Johann Gottfried (1988): Italienische Reise. Briefe und Tagebuchaufzeichnungen 17881789. Hrsg. v. Albert Meier / Heide Hollmer. München.

Herzog, Valentin: Frau Kauffmann, Goethe und Co. „,Der prüfende Blick“ von Gabrielle Alioth. In: Basler Zeitung 14.02.2007.

Katz, Gabriele (2012): Angelika Kauffmann. Künstlerin und Geschäftsfrau. Stuttgart.

Klüger, Ruth (2010): Die zehnte Muse. In: Dies.: Was Frauen schreiben. Wien. S. 151-154.

Kovalevski, Bärbel (Hrsg.) (1999): Zwischen Ideal und Wirklichkeit. Künstlerinnen der Goethe-Zeit zwischen 1750 und 1850. [Anläßlich der Ausstellung]. Ostfildern-Ruit.

Królikiewicz, Grażyna / Płaszczewska, Olga / Puchalska, Iwona / Siwiec, Magdalena (Hrsg) (2009): Literatura a malarstwo - malarstwo a literatura. Panorama myśli polskiej XX wieku. Einl. G. Królikiewicz. Kraków.

Kuhn, Nicola: Suchbild. In: Der Tagesspiegel (CH) 21.02.2007.

Kunze, Rita: Schreiben gegen die Etikette des historischen Romans. In: Mitteldeutsche Zeitung, 15.10.2008.

Lessing, Gotthhold Ephraim (2016): Laokoon oder Über die Grenzen der Malerei und Poesie. Hrsg. v. Karl-Maria Guth. Berlin. 
Lettau, Annette: Europas kultivierteste Frau. In: Focus Magazin 1998, Nr. 48, 23.11.1998, http:// www.focus.de/kultur/medien/kunst-europas-kultivierteste-frau_aid_174082.html (Zugriff am 7.04.2018).

Maierhofer, Waltraud (1997): Angelika Kauffmann. Rowohlt, Reinbek b. Hamburg.

Maierhofer, Waltraud (2012): Angelica Kauffmann Reads Goethe. Illustration and Symbolic Representation in the Göschen Edition. In: The Sophie Journal Bd. 2, Nr. 1. S. 1-34.

Mancoff, Debra N. (2012): Frauen, die die Kunst veränderten. Üb. M. Barth. München.

Obermeier, Siegfried (1987): Die Muse von Rom. Angelika Kauffmann und ihre Zeit. Frankfurt am Main.

Parton, James (1887): Angelica Kaufmann. In: Ders.: Daughters od Genius. A Series of Sketches. Philadelphia. S. 143-148.

Pender, Malcolm (2010): ,, Man kann das Gegenwärtige nicht ohne das Vergangene erkennen “. Drei historische Romane in der jüngsten Schweizer Literatur. In: Colloquia Germanica Stettinensia Nr. 18, hrsg. v. Dorota Sośnicka. Szczecin. S. 139-159.

Pender, Malcolm / Burns, Barbara (Hrsg.) (2015): Konstruktionen der Vergangenheit in der Deutschschweizer Literatur. Würzburg.

Rebmann, Jutta (2007): Angelika Kauffmann. Gefeierte Malerin im Europa des 18. Jahrhunderts. Biographischer Roman. Neuausg. München.

Rosenthal, Angela (1996): Angelika Kauffmann. Bildnismalerei im 18. Jahrhundert. Berlin.

Rytel, Elisabeth / Studer, Liliane / Stump, Doris / Widmer, Maya / Wyss, Regula (1994): Und schrieb und schrieb wie ein Tiger aus dem Busch. Über Schriftstellerinnen in der deutschsprachigen Schweiz. Zürich.

Schindler, Feli (1998): Als die Welt nach Angelika verrückt war. In: Tages-Anzeiger (CH) 19.11.2007.

Schweizerisches Institut für Kunstwissenschaft: Biografisches Lexikon der Schweizer Kunst. Unter Einschluss des Fürstentums Liechtenstein. 2 Bde. Zürich.

Segebade, Insa (2007): „, Schreiben ist Arbeit-aber es gibt keine Bessere!“ Die Schriftstellerin Gabrielle Alioth. In: TextArt (Nagel\&Kimche) 4.

Sello, Gottfried (2004): Malerinnen aus vier Jahrhunderten. Hamburg.

Senn-Schnitzler, Anna (1952): Eine unerwartete Begegnung mit Angelika Kauffmann. In: Bündner Monatsblatt: Zeitschrift für Bündner Geschichte, Landeskunde und Baukultur, H. 1. S. 24-28.

Sternberg, Alexander von (1848): Angelika Kaufmann. In: Berühmte deutsche Frauen des achtzehnten Jahrhunderts. Erster Theil. Leipzig. S. 163-200.

Sternburg, Judith von: Sich bloß nichts anmerken lassen. Angelika Kauffmanns 200. Todestag. In: Frankfurter Rundschau 5.11.2007.

Thurnher, Eugen (Hrsg., Einl.) (1966): Angelika Kauffmann und die deutsche Dichtung. Vorarlberger Schriften Bd. 10. Bregenz.

Winckelmann, Johann Joachim (1982): Winckelmanns Werke in einem Band. Hrsg. v. Helmut Holtzhauer. Berlin/Weimar.

Zima, Peter V. (2008): Der europäische Künstlerroman: von der romantischen Utopie zur postmodernen Parodie. Tübingen.

Germanica Wratislaviensia 143, 2018

(C) for this edition by CNS 